\title{
Review On: Application of Nanotechnology in Computer Science
}

\author{
Latika Susheel Chaudhary $^{1}$, Prerana Ravindra Ghatmale ${ }^{2}$, Dr. S. S. Chavan ${ }^{3}$ \\ M. Tech, Nanotechnology, Bharti Vidyapeeth Deemed University College of Engineering, Pune
}

\begin{abstract}
Nanotechnology is a new emerging technology having endless applications in the real world. It is a technology whose future is a completely uncharted; taking both science and technology to levels we never imagined. It is an interdisciplinary field having its application and development in various fields such as applied science, mechanical, electrical and many others. In recent year's nanotechnology have brought evolution in current era of various application. In this paper we highlight the role of nanotechnology in computers science and the current development, opportunities occurred in recent years. This being a review paper gives brief information about the research taken place till this date.
\end{abstract}

Keywords: nanotechnology;computing;dna computing;quantum computing;computaional nanotechnology

\section{Introduction}

Nanotechnology is the technology based on tiny things mostly comprising of nanostructures, atoms and molecules. Its scale of measurement is nanometer scale, which is one billionth of a meter which is smaller than wavelength of visible light or hundred thousand width of a human hair. It is the engineering of functional system at molecular scale. In its original sense, 'nanotechnology' refers to the projected ability to construct items from the bottom up, using techniques and tools being developed today to make complete, high performance products. The idea of nanotechnology was first proposed by a physicist named Richard Feynman in 1959. Feynman never technically used the term "nanotechnology" or "nanite" but he gave a speech called "There's Plenty of Room At the Bottom" in which he talked about how we would one day be able to manipulate atoms and molecules and actually craft them into whatever we wanted them to be. He then discussed the possibility of us, in the distant future, creating extremely small machines that would serve as tiny tools. This idea was earlier considered completely radical; we now see nanotechnology as a very real and potential technology in the near future. Nanotechnology wasn't seen as a considerable concept until the 1980s when Eric Drexler began doing research into nanotechnology, including the observation of Feynman's speech. Drexler spent countless years perfecting this concept and getting many different scientists involved with actually producing nanotechnology. It has impacts in various fields such as Computing and Data storage, Materials and Manufacturing, Health and medicine, Energy, Transportation etc. In Computing and Data storage field, it helps in development of processors with high speed, high durability, less energy consumption etc. Also helps in improvement in display and quantum technologies. In Materials and Manufacturing field, it helps in production of self healing machines, nanoscale building blocks and various materials without aid of machines. In Health and Medicine field, it helps in producing nanorobots to combat cancer cells, nanosensors for early diagnosis, etc. In Transportation field, it helps in producing light weight and low fuel consuming vehicles and also thermal and wear resistant coatings. In energy field, it provides alternative source of energy which has the capability to replace even solar energy. The basic concept that links nanotechnology to computer science just like many other applications is when materials are scaled to the nano level they develop various tunable and desirable properties such as optical, electronic, mechanical, magnetic which are otherwise absent in bulk materials. Also implied by Moore's law - which has observed that, over the history of computing hardware, the number of transistors in a dense integrated circuit has doubled approximately every two years - computer device components are being scaled down further and further as a consequence. Traditional bulkscale or micro-scale silicon-based transistors were unable to keep up with this downscaling of size, thus nanotechnology and nanomaterials came into the picture. Computer Science has large scope of application in relation with nanotechnology. This paper provides a review on the role of nanotechnology in field of computer science. The organization of paper is in following manner first part of paper is Introduction, it is followed by evolution of nanotechnology and various process involved in building up the nanoscale system, third part includes the current development of application of nanotechnology in field of computer science and lastly the challenges ,future scope ,conclusion of nanotechnology and computer science.

\section{Evolution, Technology and Application}

\section{A. Evolution of Nanotechnology}

According to Mihail Roco of the U.S. National Nanotechnology Initiative, the evolution of nanotechnology has been divided into four generations based on the products, Rocco said that the first generation of Nanotechnology began in 2000. First-generation products, also known as "passive nanostructures," are designed to perform one task such as colloids and aerosols. The second generation, which came about in 2005, is called "active nanostructures." They have a multitasking ability, and the examples are actuators and sensors. The third generation, or the era of systems of nanosystems, is expected to begin in 2010. These nanosystems will consist of thousands of interacting components like 3D networking and new hierarchical architectures, robotics, and guided assembling. The fourth generation of Nanotechnology will begin in 


\section{International Journal of Science and Research (IJSR) \\ ISSN (Online): 2319-7064}

Index Copernicus Value (2013): 6.14 | Impact Factor (2014): 5.611

around 2015 when the first integrated nanosystems are expected to be developed

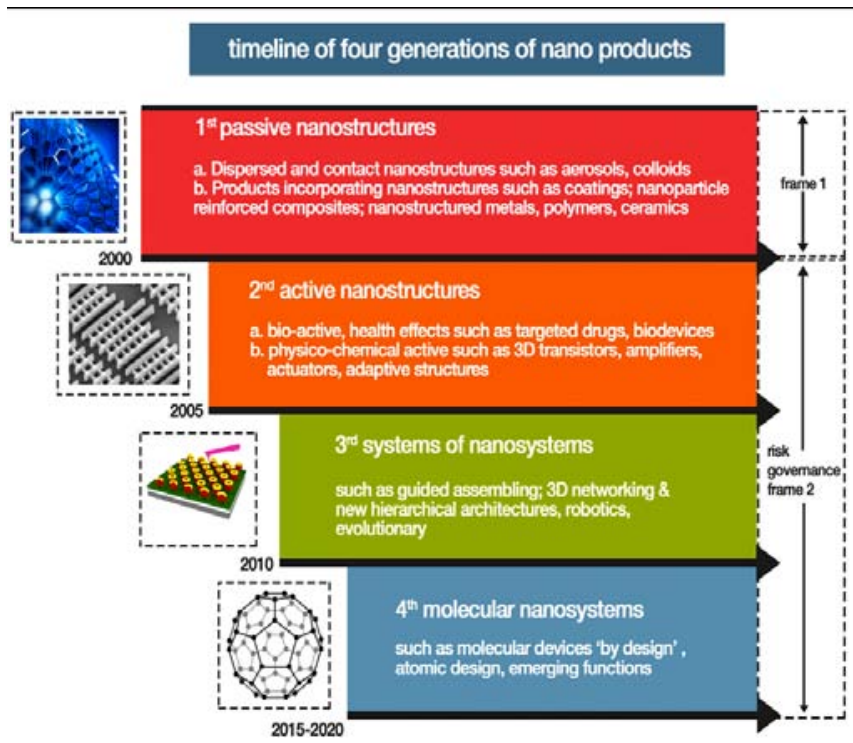

Figure 1: Four generation of Nanotechnology

\section{B. Technology and Techniques}

Nanotechnology uses the technique of Nanofabrication which helps to manipulate and integrate at atomic level and is particularly interest for computer engineer because it opens the door to super-high-density microprocessors and memory chips. It is the design and manufacture of devices with dimensions measured in nanometers. One can broadly divide various nanofabrication techniques into two categories:

- TOP-DOWN APPROACH: Top down approach seeks to create smaller devices by using larger ones to direct their assembly. The top down approach often uses the traditional micro fabrication methods in which externally controlled tools are used to cut, mill and shape materials into the desired shape and order. The most common top down fabrication technique is nano lithography. In this process required material is protected by a mask and the exposed material is etched away. Depending upon the level of resolution required for features in the final product etching of the base material can be done chemically using acids or mechanically using ultra violet light, x-rays or electron beams. This is the technique applied to manufacture computer chips.

- BOTTOM-UP APPROACH: It relies on molecular recognition and self-assembly to fabricate nanostructures out of smaller building blocks (molecules, colloids, and clusters). It has a more chemical engineering and material science flavor and relies on fundamentally different principles. A good example of this kind of approach is found in nature; all cells use enzymes to produce DNA by taking the component molecules and binding them together to make the final structure. Chemical synthesis, self-assembly, and molecular fabrication are all examples of bottom-up techniques. Self-assembly occurs when little manipulation occurs, but components spontaneously come together to form ordered nanoscale structures, for instance in the formation of nanotubes and some monolayers.

\section{Current Scenarios of Nanotechnology and Computer Science}

The impact of nanotechnology on computers has a few unique twists; which are mainly in the research and development phases. Here are examples of how nanotechnology has changed computer and its various aspect:

- Carbon Nanotube Computer: Carbon nanotubes (CNTs) are hollow cylinders composed of a single sheet of carbon atoms. It has been observed that CNT has same property as Silicon transistor and thus they act as semiconductor which makes them suitable for being used as transistor in computer chips. A team of Stanford engineers has built a basic computer using carbon nanotubes, which has the potential to make a new generation of electronic devices that run faster, and makes use of less energy, as compared with silicon chips[8]. This nanotube processor is made up of 178 transistors, each of which contains carbon nanotubes that are about 10 to 200 nanometer long. It has been reported by the they have made six versions of carbon nanotube computers, out of which one them can be connected to external hardware, and a numerical keypad that can be used to input numbers for addition[9].

- Quantum Computing: Quantum computing may well be the future of most high-end data centers. These future computers are not based upon digital 1's and 0's. Instead these future computers are based upon qubits (quantum bits). The power of magnetic forces at a subatomic scale will unleash the exponential power of future computers. By manipulating the rotation of atoms, data can be transmitted and stored at an unprecedented rate. Physicists have found a way to extend the quantum lifetime of electrons by more than 5,000 per cent. Electrons exhibit a property called 'spin' and work like tiny magnets which can point up, down or a quantum superposition of both. The state of the spin can be used to store information and so by extending their life the research provides a significant step towards building a usable quantum computer[13][14]

- Computational Nanotechnology: Nanoscale systems, though infinitesimal, are made up of thousands, even hundreds of thousands, of atoms. Thus, describing their electronic structures and dynamics requires significant theoretical skill and much computer power. It is the method to study nanoparticles using computer models to predict their behaviour and inform real life nanoparticle physics and chemistry. Computational nanotechnology is a powerful tool for understanding nanoparticle physics and chemistry. After carrying out a simulated experiment, theory is developed to explain the observed results, which is then validated by conducting a lab experiment. If the predicted results and the theoretical results agree, then the theory is accepted. Unexpected results from laboratory work can also be examined with theoretical methods, which often lead to the development of new theory. One of the example of Computational Nanotechnology is development of NanoDesign, A research group at NASA has been developing this 


\section{International Journal of Science and Research (IJSR) \\ ISSN (Online): 2319-7064}

Index Copernicus Value (2013): 6.14 | Impact Factor (2014): 5.611

software system, for investigating fullerene nanotechnology and designing molecular machines. The software architecture of NanoDesign is designed to support and enable their group to develop complex simulated molecular machines[2].

- DNA Computing: DNA computation is based on the fact that technology allows us to 'sequence' (design) single DNA strands which can be used as representations of bits of binary data. Technology also allows us to massively 'amplify' (reproduce) individual strands until there are sufficient numbers to solve complex computational problems. DNA molecule has a double helix structure composed of two sugar phosphate backbones formed by the polymerization of deoxy-ribose sugar. Placed between two backbones are pairs of nucleotides Adenine, Cytosine, Guanine and Thymine. DNA computers use single strands of DNA to perform computing operations. DNA computing focuses on the use of massive parallelism, or the allocation of tiny portions of a computing task to many different processing elements. The structure of the DNA allows the elements of the problem to be represented in a form that is analogous to the binary code structure. Trillions of unique strands of DNA are able to represent all of the possible solutions to the problem. Some scientists predict a future where our bodies are patrolled by tiny DNA computers that monitor our well-being and release the right drugs to repair damaged or unhealthy tissue.[17]

- Single Electron Transistor: The single electron transistor is made of an island connected through two tunneling junctions to a drain and a source electrode, and through a capacitor to a gate electrode. When all the biases are zero, electrons do not have enough energy to tunnel through the junction. However, if you increase the bias, but keep it less than the coulomb gap voltage, increasing the gate bias above the point of maximum slope on the coulomb staircase causes the state with one or zero excess electrons on the island to have the same energy, resulting in the coulomb barrier being removed and allowing electrons to tunnel through the junctions and between the source and the drain. The Coulomb energy is given by $\mathbf{E c}=\mathbf{e} \mathbf{2}$ / 2C, Where e is the charge on an electron and $\mathrm{C}$ is the total capacitance of the source and drain junctions and the gate capacitor. When the bias between the source and drain is greater than e/C $(e / 2 C$ across each junction), called the Coulomb gap voltage, electrons actively tunnel across the junctions, resulting in a current through the transistor independent of the gate bias. Thus Single-electron transistors (SETs) hold great promise for future nanoelectronic circuits due to their small size, low power consumption, and ability to perform fast and sensitive charge measurement.[16]

- Nanobots: Nanobots will be the next generation of nanomachines. Advanced nanobots will be able to sense and adapt to environmental stimuli such as heat, light, sounds, surface textures, and chemicals; perform complex calculations; move, communicate, and work together; conduct molecular assembly; and, to some extent, repair or even replicate themselves. Nanotechnology is the science and application of creating objects on a level smaller than 100 nanometers. The extreme concept of nanotechnology is the "bottom up" creation of virtually any material or object by assembling one atom at a time. Although nanotech processes occur at the scale of nanometers, the materials and objects that result from these processes can be much larger. Large-scale results happen when nanotechnology involves massive parallelism in which many simultaneous and synergistic nanoscale processes combine to produce a large-scale result. Nanorobots have potential applications in the assembly and maintenance of sophisticated systems. Nanorobots might function at the atomic or molecular level to build devices, machines, or circuits, a process known as molecular manufacturing. Nanorobots might also produce copies of themselves to replace worn-out units, a process called self-replication.

\section{Conclusion and Future Scope}

- Nanotechnology will be able to improve scientific exploration by far simply because nanites are so small.

- The current trends and the future development will lead to huge contribution in the field of computer science.

- Today nanotechnology being in the fourth generation of evolution is likely to show outstanding innovations in near future.

- This will increase the quality of life in our society.

\section{Acknowledgment}

We would greatfully acknowledge Kenneth Buddha Jeans for the graphical illustration in this paper denoted as Figure 1 of Four Generation of Nanotechnology and its usefulness in development of different product at nanoscle.

\section{References}

[1] Zobair Ullah," Nanotechnology and Its Impact on Modern Computer", Global Journal of Researches in Engineering General Engineering, Volume 12 Issue 4 Version 1.0 Year 2012.

[2] Boonserm Kaewkamnerdpong and Peter J. Bentley," Computer Science for Nanotechnology: Needs and Opportunities", Department of Computer Science, University College London, UK.

[3] Deepak Srivastava, Satya N. Atluri "Computational Nanotechnology: A Current Perspective”, CMES, vol.3, no.5, pp.531-538, 2002.

[4] "Computer Engineering and Nanotechnology" N.G. Rambidi, Molecular Computing, DOI 10.1007/978-3211-99699-7_2

[5] Sachin Kumar, Garima Pant, Vibhor Sharma, Pooja Bisht" Nanotechnology in Computers", International Journal of Information \& Computation Technology,ISSN 0974-2239 Volume 4, Number 15 (2014), pp. 1597-1603.

[6] Boonserm Kaewkamnerdpong, Navneet Bhalla, Peter J. Bentley "Programming Nanotechnology: Learning from Nature".

[7] www.nanowerk.com/news $2 /$ newsid=32466.php .

[8] www.technologyreview.com/s/519421/the-first-carbonnanotube-computer/.

[9] www.crnano.org/whatis.htm

[10] www.futurehumanevolution.com/nanotechnologyintroduction-series-computers. 


\section{International Journal of Science and Research (IJSR) \\ ISSN (Online): 2319-7064}

Index Copernicus Value (2013): 6.14 | Impact Factor (2014): 5.611

[11]www.nano.gov

[12] www.futureforall.org/nanotechnology/nanotechnology.h tm

[13] http://www.nanotechnow.com/news.cgi?story_id $=47080$

[14] http://www.futuretechnology500.com/index.php/futurecomputers/

[15] http://www.azonano.com/article.aspx?ArticleID=3251

[16] Alexander Coronado,"Review of quantum wires and dots in nanotechnology", 2605672 EEE-5425, Introduction to Nanotechnology Spring 2013.

[17] ISSN 2231-1297, Volume 3, Number 1 (2013), pp. 5762 (C) Research India Publications

[18] International Journal of Emerging Sciences ISSN: 22224254 1(1) April 2011 